\title{
La dictée au cours préparatoire, les conditions d'un enseignement efficace
}

Dictation in the preparatory course, the conditions for effective teaching

Jérôme Riou et Catherine Brissaud

\section{(2) OpenEdition}

Journals

Édition électronique

URL : https://journals.openedition.org/pratiques/10962

DOI : 10.4000/pratiques.10962

ISSN : 2425-2042

Éditeur

Centre de recherche sur les médiations (CREM)

Référence électronique

Jérôme Riou et Catherine Brissaud, "La dictée au cours préparatoire, les conditions d'un enseignement efficace », Pratiques [En ligne], 191-192 | 2021, mis en ligne le 15 décembre 2021. consulté le 04 janvier 2022. URL : http://journals.openedition.org/pratiques/10962 ; DOI : https:// doi.org/10.4000/pratiques. 10962

Ce document a été généré automatiquement le 4 janvier 2022.

(c) Tous droits réservés 


\section{La dictée au cours préparatoire, les conditions d'un enseignement efficace}

Dictation in the preparatory course, the conditions for effective teaching

Jérôme Riou et Catherine Brissaud

\section{Problématique et questions de recherche}

1 La dictée est un marqueur social de la maitrise de l'orthographe. En effet, les Français témoignent d'un fort engouement pour cette pratique et les médias ne s'y sont pas trompés. L'émission télévisée "Les dicos d'or" diffusée sur une chaine publique nationale de 1985 à 2005 et celle intitulée « Tous prêts pour la dictée » du 6 mai 2020 ont connu un franc succès. Elles permettent aux participants d'évaluer leur niveau en orthographe, en dénombrant les difficultés ou les pièges surmontés et en se mesurant à d'autres. Toutefois, ce caractère purement évaluatif de la dictée n'est plus représentatif de l'ensemble des formes scolaires.

2 Les enseignants n'ont jamais cessé de pratiquer la dictée, contrairement à ce que pourraient laisser penser tous ceux qui plaident en faveur du retour de la dictée à l'école. En revanche, ils ne pratiquent pas tous, ni exclusivement, la dictée dite «magistrale». En effet, cette version classique de la dictée entre désormais en concurrence avec des alternatives parfois qualifiées de dispositifs innovants qui permettent de raisonner collectivement sur le fonctionnement du système orthographique. Parmi ces dispositifs, la phrase dictée du jour et la dictée zéro faute conduisent à débattre d'arguments afin de déterminer la bonne graphie des unités linguistiques dictées (Cogis, 2005 ; Cogis et al., 2015). Ainsi, les activités s'apparentent à des résolutions de problèmes dans lesquelles les interactions didactiques prennent une place importante, pour ne pas dire essentielle. Grâce à l'émergence et l'utilisation de formes nouvelles, la dictée n'est plus seulement perçue comme un outil d'évaluation des connaissances, elle est également un moyen d'apprendre. En instaurant l'idée selon 
laquelle il est permis de douter de l'orthographe des mots, les enseignants incitent les élèves à dialoguer afin de résoudre ensemble des problèmes d'orthographe. L'institution (Ministère de l'Éducation nationale, 2017) relaie désormais les propositions des chercheurs et incite les enseignants à utiliser la phrase dictée du jour, la dictée zéro faute, la dictée sans erreur, la dictée frigo ou encore la dictée négociée. L'efficacité de ces alternatives à la dictée classique a rarement été évaluée, les études exhaustives et quantitatives de l'usage réel de la dictée dans les classes restant peu nombreuses (Sautot, 2015 ; Cogis et al., 2015). Une étude québécoise indique toutefois que les élèves de $2^{\mathrm{e}}$ cycle primaire (8-10 ans) apprenant l'orthographe grammaticale à l'aide de dictées innovantes progressent davantage que les élèves confrontés à des dictées classiques (Fischer \& Nadeau, 2014 ; Cogis et al., 2015). Plus récemment, des études françaises se sont intéressées aux effets des durées d'enseignement de la dictée sur les progrès en décodage et en orthographe des élèves de cours préparatoire (Brissaud et al., 2016 ; Goigoux, 2016 ; Riou, 2017). Leurs auteurs précisent que ces effets varient selon le niveau initial des élèves. En décodage, ceux qui obtiennent des résultats faibles et intermédiaires à l'entrée du cours préparatoire décodent mieux lorsque les durées d'enseignement de la dictée augmentent, jusqu'à environ 40 minutes par semaine. Ceux qui sont initialement forts ne bénéficient pas de cette augmentation de durée. Par ailleurs, seuls les élèves de niveau intermédiaire progressent en orthographe lorsque les durées d'enseignement s'accroissent, dans l'intervalle de données relevées, c'est-à-dire jusqu'à 71 minutes par semaine.

Les progrès des élèves diffèrent donc selon leur niveau à l'entrée du cours préparatoire et les compétences évaluées. Sous l'effet de l'enseignement de la dictée, certains mémorisent mieux les correspondances entre les lettres et les sons alors que d'autres commettent moins d'erreurs d'orthographe. Ces résultats sur les durées d'enseignement nous conduisent à nous interroger sur la mise en œuvre effective des activités d'écriture sous la dictée. Ils nous invitent aussi à réinterroger le rapport entre les objectifs d'enseignement visés par cette pratique et les compétences réellement développées.

4 Nous nous appuierons sur les enregistrements vidéo collectés lors de la recherche Lire et écrire (Goigoux, 2016) pour identifier ce qui, dans les pratiques des professeurs, peut conduire à des acquisitions différenciées. Plus précisément, nous essaierons de repérer des caractéristiques de l'efficacité des pratiques d'enseignement dans le type d'activité proposé, les modalités d'organisation pédagogique et les interactions didactiques. Nous nous intéresserons notamment à la nature des tâches prescrites, aux unités linguistiques dictées et au traitement des réponses des élèves. Nous nous intéresserons également au rôle de la morphologie dans la construction des compétences en orthographe. Nous concevrons des modèles multiniveaux pour évaluer les effets des durées d'enseignement de la morphologie sur les progrès des élèves.

\section{Pratiquer la dictée dans un système orthographique complexe}

Le système orthographique du français est à la fois phonographique et sémiographique (Ducard et al., 1995 ; Cogis, 2005). Il repose essentiellement sur la mise en correspondance de phonèmes et de graphèmes, mais également sur l'emploi de marques morphologiques qui résultent de l'application de normes lexicales et 
grammaticales. Les marques morphologiques, qu'elles soient dérivationnelles ou flexionnelles sont le plus souvent sans correspondant phonologique, ce qui rend le travail d'encodage difficile (Brissaud \& Cogis, 2011). Les liaisons permettent d'identifier certaines marques, à condition de disposer d'une maitrise suffisante de la chaine parlée. Les activités d'encodage mobilisent donc un ensemble de connaissances ayant trait à la fois aux principes phonographique et sémiographique de l'orthographe. Selon la nature des tâches prescrites aux élèves et les modalités de résolution proposées par les professeurs, les connaissances mobilisées peuvent sensiblement varier d'une séance à l'autre et, à fortiori, d'une classe à l'autre.

6 L'oral et l'écrit sont reliés par un code, et c'est cette même relation qui unit l'orthographe et la lecture. Ces deux apprentissages ne sont cependant pas équivalents. Sur un plan strictement linguistique, l'écriture est plus difficile que la lecture. Souvent, les graphèmes ne renvoient qu'à une seule prononciation alors que les phonèmes peuvent être transcrits par plusieurs phonogrammes. Par exemple, il est plus difficile de choisir le bon phonogramme pour /o/ parmi les trois possibilités qu'offre sa transcription (o, au, eau) que de trouver le correspondant phonologique de l'un des graphèmes en question. En effet, seules un peu plus de $50 \%$ des formes graphiques peuvent être prédites à partir de leur forme phonologique (Véronis, 1988 ; Ziegler et al., 1996). Il est aussi plus difficile d'effectuer des marquages morphologiques non sonores que d'identifier des lettres muettes et de ne pas les prononcer, notamment lorsque celles-ci occupent une position finale dans les mots. Si un élève a compris que le graphème "é ", mais aussi " és ", "ée ", "ées ", se prononçaient /e/, il pourra lire l'ensemble des séquences orthographiques correspondantes. En revanche, il lui sera plus difficile de trouver l'orthographe correcte de /e/ en finale d'un mot (SprengerCharolles \& Colé, 2013).

7 L'orthographe française est donc plus prévisible ou «consistante» dans le sens de la lecture que dans celui de l'écriture, autrement dit de l'écrit vers l'oral que de l'oral vers l'écrit. Le fait que la lecture soit à priori d'un accès plus facile que l'écriture ne signifie pas pour autant que la pratique du décodage doit précéder celle de l'encodage. Les résultats d'une étude d'envergure récente conduite au cours préparatoire tend plutôt à prouver le contraire (Goigoux, 2016). D'autres études soulignent l'importance d'encoder tôt, dès l'école maternelle (Bégin et al., 2005; Morin \& Montésinos-Gelet, 2007 ; Sénéchal et al., 2012 ; Alves Martins et al., 2013 ; Alves Martins et al., 2014) et rappellent l'intérêt précoce des élèves pour le fonctionnement des systèmes d'écriture (Pacton, 2008).

\section{La dictée, une activité d'encodage spécifique}

8 Au cours de leurs premiers essais d'écriture sous la dictée, les élèves qui ont compris le principe alphabétique, autrement dit le fait que l'écrit code la parole, repèrent des configurations sonores et établissent des correspondances avec des configurations de lettres. Ils segmentent les énoncés en unités linguistiques de tailles variables comportant un ou plusieurs phonèmes. Ils maintiennent cette segmentation en mémoire et la transcrivent pas à pas. Au cours de cette phase de médiation phonologique, ils mettent en relation des phonèmes et des graphèmes, des configurations sonores supérieures au phonème et des séquences orthographiques (Fayol \& Jaffré, 2014). Ils procèdent parfois par analogie en s'appuyant sur leurs 
compétences lexicales ou en se référant à des écrits situés dans leur environnement proche.

L'écriture sous la dictée est une activité d'encodage consistant à assembler des lettres ou des groupes de lettres afin de produire un écrit qui respecte les normes orthographiques d'un système d'écriture donné. À ce titre, elle appartient à un ensemble plus vaste qui regroupe d'autres activités de même nature. Au cours préparatoire, les professeurs prescrivent quatre types de tâches d'encodage. Ils demandent aux élèves : 1) d'écrire sous la dictée ;2) de produire seuls des écrits ; 3) de produire des syllabes ou des mots à l'aide d'étiquettes préimprimées; 4) de préciser l'écriture de syllabes ou de mots pendant l'activité de dictée à l'adulte. Les élèves de cours préparatoire résolvent essentiellement des tâches d'écriture sous la dictée et de production autonome d'écrits, celles-ci représentant plus de $85 \%$ des durées d'encodage (Goigoux, 2016).

L'écriture sous la dictée est une activité spécifique dans la mesure où les élèves transcrivent les unités linguistiques que le professeur choisit. Elle est notamment très différente de la dictée de syllabes et de mots à l'aide d'étiquettes préimprimées sur lesquelles les lettres et les syllabes sont correctement orthographiées. En effet, cette dernière repose exclusivement sur l'analyse de composantes sonores et ne nécessite pas de transcrire d'éventuelles marques morphologiques. La tâche de l'élève consiste à identifier les lettres et les syllabes avant de composer avec elles. La lecture devient alors un préalable à l'écriture, ce qui n'incite pas les élèves à interroger la norme orthographique.

\section{Quelles implications pour la classe?}

11 Au début de l'apprentissage de la lecture, l'étude des relations phonographiques occupe une place centrale car «lire, c'est d'abord décoder " (Ministère de l'Éducation nationale, 2019, p. 78). Pendant cette période, il est donc nécessaire d'enseigner l'écriture en portant une attention particulière aux procédures des élèves dans les situations d'encodage, en n'induisant pas une décomposition systématique des énoncés en phonèmes. En effet, cette démarche conduirait au développement de "stratégies d'encodage inverses de celles mobilisées pour le décodage " (Chiss \& David, 2011, p. 202), et formerait davantage à la maitrise du code alphabétique qu'à celle de l'orthographe. Or, «la fonction majeure de l'orthographe n'est pas de noter avec précision la prononciation » mais de chercher «à saisir le principe d'une structure formelle capable de véhiculer du sens » (Jaffré, 2005, p. 24). Des connaissances autres que les relations entre les graphèmes et les phonèmes sont indispensables à l'écriture, elles sont morphologiques, grammaticales et lexicales. Ces connaissances jouent un rôle essentiel au moment de choisir le bon phonogramme, le bon affixe, une séquence orthographique, ou d'appliquer des règles d'accords.

12 L'écriture sous la dictée correspond à la transcription d'un matériau phonique, celui que le professeur énonce, en un matériau graphique, celui que les élèves produisent. La forme «traditionnelle » de l'écriture sous la dictée et sa fonction évaluative a vu naitre de nombreuses variantes ${ }^{1}$ qui présentent autant de modalités de mise en œuvre que de finalités. En effet, la dictée ne sert plus seulement à déterminer le niveau de compétences des élèves en orthographe à un instant " $t$ ", elle contribue aux apprentissages. L'atelier de négociation graphique et la phrase dictée du jour, inspirés 
des travaux de E. Ferreiro et de ceux plus anciens de J. Piaget et L. Vygotski, sont deux dispositifs d'enseignement qui illustrent ce changement de paradigme (Brissaud \& Cogis, 2011). Ils ont pour but de faire émerger les représentations des élèves et de les discuter publiquement afin de mieux appréhender le fonctionnement du système orthographique. Au cours des interactions didactiques, les élèves sont invités à justifier leurs choix, ce qui les amène à conduire une réflexion sur la langue. Cette activité métalinguistique est de nature à développer leurs compétences orthographiques.

Face à la multiplicité des modalités et des finalités de résolution des tâches d'écriture sous la dictée, il convient de ne pas s'en tenir aux durées d'enseignement et d'analyser plus finement les pratiques des enseignants. L'une des entrées propices à cette analyse est celle qui consiste à s'intéresser aux unités linguistiques mobilisées par les tâches administrées aux élèves. Les dictées de syllabes induisent prioritairement l'étude des correspondances graphophonémiques. En effet, la tâche des élèves consiste à produire des syllabes phonologiquement compatibles avec l'énoncé du professeur. Il n'est donc pas exclu que certaines de ces productions ne correspondent à aucune syllabe du français (/ka/ transcrit " qua », par exemple), bien que le nombre de celles-ci, environ 6000 (Fayol et Jaffré, 2008, p. 87), limite ce phénomène. En revanche, la probabilité est plus forte que les élèves produisent des syllabes peu fréquentes en français, qui entrent dans la composition de mots qu'ils méconnaissent encore, et qui ne leur soient donc pas très utiles à ce moment de leur scolarité.

14 À la différence des syllabes, les mots sont des unités linguistiques signifiantes qui peuvent figurer dans le répertoire lexical des élèves et potentiellement être associés à des sonorités ou des images mentales. Le triptyque constitué par la phonologie, l'orthographe et la sémantique, quand il est efficient, est une aide précieuse à la lecture et à l'écriture. En outre, il est intéressant que les élèves puissent identifier la fonction grammaticale des mots afin de sélectionner des marques morphologiques adaptées. Les mots gagnent donc à être énoncés dans des phrases, inscrits dans un champ lexical et sémantique, afin que le scripteur puisse choisir la bonne orthographe. L'utilisation de ces procédés est indispensable dès lors que les mots dictés présentent un ou plusieurs homophones hétérographes (ver, vert, vers, vair et verre, par exemple). En effet, les homophones hétérographes sont «morphologiquement motivés " (Jaffré, 2006, p. 33), ils sont transcrits par des phonogrammes différents ou portent des marques dérivatives qui ont essentiellement une fonction sémantique (Bégin et al., 2010). La plupart des mots étant plurimorphémiques ${ }^{2}$ (Rey-Debove, 1984), la connaissance des affixes (préfixes et suffixes) est déterminante afin de produire une orthographe normée.

Les dictées de propositions ou de phrases vont conduire à l'emploi de suffixes flexionnels marquant soit le genre et le nombre des noms et des adjectifs, soit le temps, la personne et le nombre des verbes. Les mots fléchis qui résultent de l'application de ces règles d'accord ont principalement une fonction syntaxique (Bégin et al., 2010). Le texte invite le scripteur à porter une attention particulière à l'orthographe des reprises anaphoriques et des accords qu'elles engendrent, autrement dit à prendre en compte les relations interphrastiques. Plus les unités linguistiques dictées sont grandes, de la syllabe au texte, plus la morphologie s'impose comme une composante essentielle de la maitrise de l'orthographe. 


\section{Méthodologie}

16 Afin d'étudier les pratiques d'enseignement de la dictée, nous avons procédé à une analyse en trois temps. Tout d'abord, nous avons déterminé le niveau d'efficacité en orthographe des 131 classes observées dans le cadre de l'étude Lire et Écrire. Pour cela, nous avons évalué les progrès des élèves au cours de l'année scolaire en leur demandant d'écrire leur prénom, les mots isolés "lapin », " rat », « éléphant » et la phrase « Tom joue avec le rat. ». Ces épreuves ont été administrées au début et à la fin de l'année de cours préparatoire. Les épreuves de fin d'année ont été complétées par la dictée d'une phrase comportant des marques du pluriel, à savoir «Les lapins courent vite.». Nous avons traité les données au moyen d'analyses de régression multiple dont le principe repose sur l'explication de la variété des performances des élèves. Nous avons donc conçu des modèles statistiques intégrant les niveaux de performance initiaux et finaux des élèves, ainsi que des caractéristiques qui leur sont propres, c'està-dire leur âge, leur genre, le milieu social auquel ils appartiennent et la (ou les) langue(s) parlée(s) à la maison. Ainsi, dans un premier modèle, les performances finales en orthographe ont constitué les variables dépendantes dont nous avons cherché à expliquer la variété. Les scores aux différentes épreuves n'ayant ni le même total, ni le même barème, nous les avons standardisés afin de les comparer. Par conséquent, nous avons rendu la moyenne de chaque épreuve égale à 0 et l'écart-type égal à 1 . Dans un deuxième modèle, nous avons introduit l'ensemble des classes en variables muettes, sauf la classe 120 choisie arbitrairement comme référence, de manière à évaluer l'effet de l'appartenance à une classe. En raisonnant à niveau initial des élèves donné et caractéristiques individuelles données, nous avons identifié des différences interclasses. Nous avons ainsi pu ordonner les 131 classes de l'étude Lire et Écrire selon leur efficacité en orthographe (cf. annexe 1). Nous avons alors choisi de nous intéresser aux 15 classes les plus efficaces et aux 15 classes les moins efficaces en orthographe, en supposant que l'étude des pratiques de classes pourraient expliquer les différences d'efficacité.

Puis, nous avons recherché les suites de tâches relevées par les enquêteurs de l'étude Lire et Écrire (Goigoux, 2016) lors des dictées et les enregistrements filmés correspondants. Nous nous sommes appuyés sur les emplois du temps hebdomadaires des enseignants, qui ont été observés pendant trois semaines, aux mois de novembre, mars et mai. Nous avons choisi d'étudier les séances du mois de mars parce qu'elles sont susceptibles de révéler de profondes disparités entre certains enseignants qui font écrire les élèves sous la dictée depuis le début de l'année scolaire, et d'autres qui préfèrent attendre que ces derniers aient acquis suffisamment de connaissances sur le fonctionnement de la langue. Cette période de l'année nous a donc semblé la plus propice à l'observation de différences interclasses et présente, en outre, la durée moyenne des tâches de dictée (E4) ${ }^{3}$ la plus élevée (voir infra la partie Résultats). Seule la moitié des classes disposait d'un enregistrement, tous n'étaient pas disponibles. Nous avons donc analysé les séances de dictée de 13 classes, 7 parmi les plus efficaces en orthographe (classes 61, 57, 88, 78, 26, 63 et 49), et 6 parmi les moins efficaces (classes $58,119,104,116,92$ et 20). Pour chacune des séances, nous avons analysé la succession de tâches prescrites aux élèves, leur durée, les unités linguistiques travaillées, les modalités de traitement des réponses des élèves et l'organisation pédagogique. Nous avons comparé les pratiques d'enseignement les unes aux autres afin d'identifier des 
caractéristiques communes à celles qui sont efficaces et à celles qui le sont moins. Enfin, nous avons complété nos données par le calcul des durées moyennes d'enseignement consacrées à la morphologie, et nous avons évalué les effets de ces durées sur les apprentissages des élèves en orthographe à l'aide de modèles d'analyse statistique multiniveaux (Bressoux, 2010). Nous avons ajouté les caractéristiques des classes aux modèles décrits plus hauts.

\section{Résultats}

\section{Durée consacrée à l'écriture sous la dictée}

Au mois de mars, les 15 classes les plus efficaces en orthographe consacrent 23 minutes par semaine en moyenne à la résolution de tâches d'écriture sous la dictée (E4) (voir tableau 1). Cette moyenne est identique à celle des 131 classes de l'étude Lire et Écrire (Goigoux, 2016). Les 15 classes les moins efficaces en orthographe y consacrent seulement une minute de moins par semaine. Les durées moyennes d'enseignement masquent toutefois une grande hétérogénéité de pratiques. En effet, certaines classes ne pratiquent pas du tout l'écriture sous la dictée, d'autres y consacrent plus d'une heure par semaine, qu'elles appartiennent au groupe des classes les plus efficaces ou des classes les moins efficaces. Ainsi, nous n'identifions pas de différence nette en comparant les deux échantillons du point de vue des durées d'enseignement, ce qui invite à analyser plus finement les pratiques.

\begin{tabular}{|l|l|l|l|l|l|}
\hline $\begin{array}{l}\text { Efficacité } \\
\text { (Rang) }\end{array}$ & $\begin{array}{l}\text { Identifiants } \\
\text { classe }\end{array}$ & $\begin{array}{l}\text { Écriture sous la } \\
\text { dictée (E4) en } \\
\text { minutes }\end{array}$ & $\begin{array}{l}\text { Efficacité } \\
\text { (Rang) }\end{array}$ & $\begin{array}{l}\text { Identifiants } \\
\text { classe }\end{array}$ & $\begin{array}{l}\text { Eriture sous la } \\
\text { dictée (E4) en } \\
\text { minutes }\end{array}$ \\
\hline 1 & 8 & 0 & 116 & 19 & 18 \\
\hline 2 & 61 & 41 & 117 & 90 & 5 \\
\hline 3 & 57 & 71 & 118 & 71 & 77 \\
\hline 4 & 88 & 6 & 119 & 31 & 17 \\
\hline 5 & 78 & 33 & 120 & 54 & 70 \\
\hline 6 & 23 & 0 & 121 & 39 & 0 \\
\hline 7 & 26 & 31 & 122 & 58 & 13 \\
\hline 8 & 63 & 28 & 123 & 62 & 26 \\
\hline 9 & 5 & 37 & 124 & 56 & 13 \\
\hline 10 & 95 & 15 & 125 & 81 & 4 \\
\hline 11 & 85 & 0 & 126 & 119 & 14 \\
\hline 12 & 27 & 21 & 127 & 104 & 12 \\
\hline 13 & 99 & 12 & 128 & 116 & 4 \\
\hline 14 & 53 & 24 & 29 & 92 & 61 \\
\hline 15 & 49 & 23 & 20 & 24 \\
\hline Durée moyenne & & 129 & 24 \\
\hline
\end{tabular}


Les séances de dictée des classes les plus efficaces en orthographe que nous avons observées durent de 10 à 50 minutes (voir annexe 3). Elles se composent essentiellement de tâches d'écriture sous la dictée (E4) mais pas seulement. Celles-ci alternent parfois avec la dictée de lettres à l'adulte (E6), une autre tâche d'encodage, la mise en relation de graphèmes et de phonèmes (PG4), l'étude de la morphologie (EL3) ou des temps de corrections des écrits produits (E9). Ces tâches portent le plus souvent sur la syllabe et le mot, plus rarement sur la proposition et la phrase, la lettre étant parfois utilisée par les élèves pour dicter des syllabes à l'enseignant.

Les séances de dictée des classes les moins efficaces en orthographe durent entre 10 et 25 minutes (voir annexe 4). Le rythme de travail est parfois lent : il faut par exemple 6 minutes pour écrire et corriger deux syllabes dans une classe, jusqu'à 25 min pour écrire et corriger 4 mots incluant le son de la semaine dans une autre. Elles se composent essentiellement de tâches d'écriture sous la dictée de syllabes et de mots, plus rarement de phrases. La variété porte plutôt sur les unités linguistiques dictées et quand il y a successivement écriture de syllabes puis de mots, les syllabes choisies sont rarement en lien avec les mots dictés ensuite; les enseignants donnent d'ailleurs parfois l'impression d'improviser le choix des syllabes. Dans deux des classes observées en mars, c'était visiblement la première dictée qui était proposée aux élèves (classes 116 et 20).

\section{La nature des dictées}

21 Au mois de mars, les dictées sont soit très homogènes sur le plan linguistique, soit de difficulté croissante. En effet, certains enseignants dictent uniquement des syllabes, des mots ou des groupes nominaux. D'autres proposent des syllabes puis des mots, des mots puis des phrases, ou encore des syllabes puis des mots et des phrases. Par ailleurs, les enseignements reposent parfois sur l'étude d'un phonème, mettant ainsi en relation la lecture et l'orthographe. Parmi les dictées observées dans les classes les plus efficaces, l'une d'elles illustre à la fois cette relation et la notion de progressivité. En effet, l'enseignante de la classe 26 propose aux élèves de produire des syllabes avec le graphème " $\mathrm{g}$ " prononcé $[\mathrm{g}]$ et d'identifier les cas dans lesquels le " $\mathrm{g}$ " doit être ou non suivi d'un « $u$ » pour produire le bon son. Elle demande aux élèves d'écrire les syllabes /ga/, /go/, /gy/, /gu/, /gə/, /gi/, les groupes nominaux /lagar/, /ləgato/, / œ̃legym/, /labag/, /lagitar/, /ləgidõ/, les mots /grã/, /grãd/ et la phrase/ ləSevalgalopdãləpre/.

Au mois de mars, la dictée est rarement utilisée pour étudier la morphologie. Seule une enseignante des classes les plus efficaces propose aux élèves d'écrire des groupes nominaux au pluriel, ceux-ci se composant d'un déterminant numéral et d'un nom commun. Dans l'exercice proposé, les pluriels sont marqués par la lettre «s", le morphème de pluriel le plus fréquent en français. Dans d'autres classes, le « $\mathrm{e}$ » muet est évoqué à l'oral en tant que marque du féminin. Toutefois, cette mise en relation entre un graphème muet et sa valeur sémantique ne renvoie ni à un affichage collectif ni à des mots référents, ce qui laisse supposer que la notion en question n'a pas été institutionnalisée. Par ailleurs, la marque du féminin est parfois indument invoquée pour justifier la présence de la lettre « e » à la fin des mots : « Au bout du mot "tortue", je vous le dis, on met un "e". C'est féminin, on met un "e" " (extrait des interactions didactique entre l'enseignante de la classe 63 et ses élèves). 
23 Les enseignants des classes les plus efficaces en orthographe dictent le plus souvent le même type d'unités linguistiques que les enseignants des classes qui le sont moins. Toutefois, leurs propositions sont plus structurées et probablement mieux préparées. En effet, ils laissent planer peu de doutes sur leurs intentions. Ils souhaitent que les élèves forment des syllabes avec un phonème récemment étudié en classe, utilisent le phonème en question ou les syllabes formées pour produire des mots, éventuellement des phrases. Certains d'entre eux utilisent les séances de dictée pour étudier la morphologie (classe 78) ou réviser des connaissances acquises antérieurement (classe 57). Quelles que soient les activités proposées, l'ensemble forme le plus souvent un tout cohérent. Ce n'est pas le cas des séances de dictée des enseignants des classes les moins efficaces en orthographe. Ceux-ci mêlent parfois la dictée et la production écrite (classe 58), les syllabes et les mots monosyllabiques, et visent la maitrise de compétences remarquables $^{4}$ (classe 104). Ils dictent des mots sans lien entre eux ou avec l'enseignement du code alphabétique et les segmentent différemment à l'écrit et à l'oral (classe 20). Ils demandent aux élèves de produire toutes les graphies plausibles d'une syllabe sans renvoyer à la norme et sans identifier les graphies les plus fréquentes en français (classe 58).

\section{Les modalités de résolution des tâches de dictée}

24 Les enseignants des classes les plus efficaces assurent un guidage progressif de la résolution des tâches d'écriture sous la dictée. Ils proposent à leurs élèves de réfléchir collectivement aux différentes graphies possibles, autrement dit négocient avec eux l'écriture des syllabes, des mots ou des phrases. Ils effectuent très majoritairement une correction immédiate des différentes propositions orthographiques, après chaque unité linguistique dictée. Lorsque le contexte de travail ne permet pas de fonctionner ainsi, en présence d'un double niveau par exemple, ils utilisent d'autres modalités. Dans la classe 78, par exemple, les élèves disposent d'images qui représentent les groupes nominaux (déterminant + nom) à orthographier. L'enseignante effectue une résolution au moins partielle des tâches préalablement à l'écriture, en prenant soin de donner aux élèves des indications utiles à la réussite de l'exercice et de discuter leurs différentes propositions. Dans ce cas précis, la négociation graphique s'opère donc en amont de l'écriture pour l'ensemble de la dictée.

25 En apparence, les façons de faire des enseignants des classes les moins efficaces sont proches de celles des enseignants les plus efficaces. Ils proposent des aides aux élèves et leur rappellent où trouver les outils dont ils ont besoin, alternent les phases de travail individuelles et les corrections collectives. Les nuances sont à chercher dans la prise en compte des réponses des élèves, dans l'accueil de l'erreur et son traitement jusqu'à la résolution de la situation proposée. On observe parfois une forme d'impatience qui conduit à ne pas laisser les élèves argumenter sur leurs choix d'écriture, débattre de ces arguments. L'enseignant se dit détenteur du savoir (« moi, je dis non », enseignant de la classe 104), ne sollicite pas les élèves et ne cherche pas à comprendre ce qui a motivé la réponse de l'un d'entre eux. Dans la classe 20, on note parfois une forme d'agacement qui n'est pas de nature à favoriser l'engagement des élèves dans la résolution des tâches proposées. Le rôle de la lettre « e » n'est pas discuté pendant la segmentation des mots «cabane » et « robe ». Enfin, dans la classe 92, 8 minutes sont consacrées à la manière 
dont il faut s'y prendre pour écrire une syllabe. Peut-être que ce temps aurait pu être écourté au profit d'un temps d'écriture pour les élèves.

\section{Les durées d'enseignement de la morphologie}

La durée moyenne d'enseignement de la morphologie (EL3) des 131 classes de l'étude Lire et Écrire est d'environ 11 minutes par semaine au mois de mars et un peu moins de 10 minutes sur l'année. Celle des classes les plus efficaces est de 15 minutes par semaine au mois de mars et sur l'année, celle des classes les moins efficaces de 6 minutes par semaine au mois de mars et seulement 4 minutes sur l'année (voir tableau 2 ci-dessous). La différence est donc nette entre les deux échantillons que nous avons constitués.

\begin{tabular}{|c|c|c|c|c|c|c|c|}
\hline \multirow{2}{*}{ Eff. (Rang) } & \multirow{2}{*}{ Id. classe } & \multicolumn{2}{|c|}{ Tâches de morphologie } & \multirow{2}{*}{ Eff. (Rang) } & \multirow{2}{*}{$\begin{array}{l}\text { Id. } \\
\text { classe }\end{array}$} & \multicolumn{2}{|c|}{$\begin{array}{l}\text { Tâches de } \\
\text { morphologie }\end{array}$} \\
\hline & & EL3 mars & $\begin{array}{l}\text { EL3 } \\
\text { moyen }\end{array}$ & & & $\begin{array}{l}\text { EL3 } \\
\text { mars }\end{array}$ & $\begin{array}{l}\text { EL3 } \\
\text { moyen }\end{array}$ \\
\hline 1 & 8 & 0 & 22 & 116 & 19 & 0 & 0 \\
\hline 2 & 61 & 28 & 35 & 117 & 90 & 11 & 8 \\
\hline 3 & 57 & 11 & 13 & 118 & 71 & 0 & 0 \\
\hline 4 & 88 & 4 & 1 & 119 & 31 & 0 & 4 \\
\hline 5 & 78 & 24 & 14 & 120 & 54 & 4 & 1 \\
\hline 6 & 23 & 10 & 18 & 121 & 39 & 0 & 0 \\
\hline 7 & 26 & 0 & 7 & 122 & 58 & 4 & 3 \\
\hline 8 & 63 & 6 & 6 & 123 & 62 & 0 & 0 \\
\hline 9 & 5 & 0 & 8 & 124 & 56 & 15 & 8 \\
\hline 10 & 95 & 0 & 0 & 125 & 81 & 0 & 3 \\
\hline 11 & 85 & 24 & 40 & 126 & 119 & 5 & 2 \\
\hline 12 & 27 & 4 & 9 & 127 & 104 & 15 & 5 \\
\hline 13 & 99 & 36 & 15 & 128 & 116 & 12 & 4 \\
\hline 14 & 53 & 44 & 18 & 129 & 92 & 25 & 17 \\
\hline 15 & 49 & 29 & 12 & 130 & 20 & 3 & 4 \\
\hline \multicolumn{2}{|c|}{$\begin{array}{llr}\text { Durées } & \text { moyennes } & \text { des } \\
\text { classes les plus } \\
\text { efficaces }\end{array}$} & 15 & 15 & \multicolumn{2}{|c|}{$\begin{array}{l}\text { Durées moyennes des } \\
\text { classes les moins } \\
\text { efficaces }\end{array}$} & 6 & 4 \\
\hline
\end{tabular}

Une seconde analyse fondée sur la conception de modèle statistiques multiniveaux (Bressoux, 2010) nous a permis de mettre en évidence un effet significatif et positif des durées d'enseignement de la morphologie sur les performances des élèves en orthographe (voir annexe 5). Il s'agit d'un effet linéaire moyen, autrement dit un effet qui s'intensifie lorsque la durée d'enseignement augmente $(p<0.01)$. Il semblerait toutefois qu'il y ait un léger infléchissement de cet effet au-delà de 34 minutes par 
semaine (effet quadratique $; \mathrm{p}<0.01 ; \mathrm{p}<0.05$ ), ce qui concerne un petit nombre de classes qui pratiquent beaucoup l'étude de la morphologie. Dans les classes efficaces, les élèves bénéficient donc de durées d'enseignement plus élevées qui contribuent à la maitrise de l'orthographe.

\section{Discussion}

La comparaison de deux échantillons de classes, les unes très efficaces en orthographe, les autres sensiblement moins, a permis de souligner quelques éléments de convergence et quelques points de divergence susceptibles d'influencer les progrès des élèves de cours préparatoire. Ce sont ces éléments qui seront au cœur de la réflexion des paragraphes qui suivent.

\section{Les durées d'enseignement}

D'après les résultats de notre étude, les séances de dictée ne semblent pas être le lieu privilégié de la résolution de tâches de morphologie. En effet, seules quelques-unes ont été identifiées lors des observations filmées et elles ne représentent qu'une infime partie des durées moyennes hebdomadaires consacrées à la morphologie. Par ailleurs, il n'existe pas de séances spécifiques dédiées à cet enseignement au cours préparatoire (Goigoux, 2016). Les tâches de morphologie sont donc disséminées dans les séances qui relèvent de la discipline "français", leur nombre et leur durée variant considérablement d'une classe à l'autre. Les enseignants des classes les plus efficaces en orthographe consacrent plus de temps à la morphologie que ceux des classes les moins efficaces, ce qui conduit les élèves à mieux maitriser l'orthographe. Ces résultats s'inscrivent dans le droit fil des recherches qui soulignent l'étroite relation que la maitrise des compétences de lecture et d'écriture entretient avec une bonne compréhension du fonctionnement du système orthographique (Castles et al., 2018).

Les effets des durées d'enseignement de la morphologie ont été évalués indépendamment des pratiques pédagogiques mises en œuvre. Cela ne signifie pas pour autant qu'il faille s'interdire d'interroger les pratiques, ne serait-ce que pour expliquer pourquoi certaines classes peu efficaces consacrent un temps suffisant à l'enseignement de la morphologie et pourquoi d'autres sont efficaces alors qu'elles y consacrent peu de temps. L'absence ou le surcroit d'efficacité en orthographe dépend d'autres durées d'enseignement, notamment le temps passé à écrire sous la dictée ou à produire des écrits de façon autonome (Riou, 2017), et probablement aussi d'autres facteurs tels que la nature des unités linguistiques dictées, l'organisation et la cohérence des activités proposées, le statut accordé à l'erreur et les opportunités offertes aux élèves de développer leur vigilance orthographique.

31 Parmi les nombreuses questions qui restent en suspens, celle de la valeur de la lettre «e » est problématique pour l'enseignement de l'orthographe. En effet, les enseignants lui attribuent souvent à tort une signification de genre, en l'occurrence celle du féminin. Or, en français, 47 \% des mots qui possèdent un « $\mathrm{e}$ » en finale et dont le genre est exclusif, autrement dit exclusivement du masculin ou du féminin, sont masculins (Séguin, 1974). Le «e » final n'étant pas une marque générale du féminin, le genre ne peut servir à justifier sa présence. Il faut notamment distinguer les marques effectives du féminin qui ne se font pas sentir à l'oral, dans « jolie » et « amicale » par exemple, 
des marques graphiques de rappel attribuées au masculin (Catach, 2012). La lettre «e » de "grande" rend audible la consonne qui la précède, elle est par conséquent diacritique.

\section{Les choix didactiques et pédagogiques}

32 Au début du cours préparatoire, les unités linguistiques privilégiées par les enseignants sont la syllabe et le mot (Goigoux, 2016). La proposition et la phrase apparaissent plus tardivement, le texte n'étant souvent proposé qu'à partir du cours élémentaire première année, sauf dans le cas de l'emploi de modalités pédagogiques particulières telle que la dictée à l'adulte. De ce point de vue, il n'y a pas de grande différence entre les classes les plus efficaces en orthographe et celles qui le sont moins. Toutefois, la dictée de syllabes n'induit pas les mêmes procédures de résolution que la dictée de mots de la part de l'élève et le même traitement des réponses de la part de l'enseignant. Dès lors qu'un phonème peut être codé par plusieurs phonogrammes, la syllabe offre plusieurs transcriptions possibles, certaines étant peu fréquentes voire absentes du répertoire syllabique du français («qo» ou «kra», par exemple). Il revient alors à l'enseignant d'accepter ou non des réponses qui correspondent à des «pseudosyllabes ». Le mot, quant à lui, renvoie à des morphèmes lexicaux ou grammaticaux dont l'écriture est normée. Les élèves peuvent donc utiliser des outils de référence pour retrouver son orthographe s'ils ne l'ont pas encore mémorisée. Par ailleurs, la mise en contexte d'un mot permet d'identifier son rôle syntaxique, de s'intéresser à ses variations ou de lever un doute orthographique s'il est polysémique.

Finalement, la dictée de syllabes incite les élèves à produire une association de graphèmes dont la prononciation est phonologiquement plausible. Elle développe donc davantage des compétences de maitrise des relations graphophonémiques que la mémorisation de graphies normées, en particulier si l'enseignant réclame une décomposition systématique des syllabes en phonèmes. Ainsi, elle détourne l'activité de son objectif premier qui est de faire apprendre l'orthographe, et favorise d'abord la construction de compétences de décodage. Ceci explique probablement pourquoi l'augmentation des durées d'enseignement de l'écriture sous la dictée permet aux élèves initialement faibles d'obtenir de meilleurs résultats en décodage et ne les aide pas davantage en orthographe (Riou, 2017). En revanche, lors d'une dictée de mots, les connaissances sur le code sont au service de la résolution d'une tâche d'orthographe, notamment lorsque le mot est mis en contexte dans une phrase à l'oral.

L'analyse de la mise en œuvre des séances de dictée soulève une autre question importante, celle de la segmentation syllabique utilisée par les enseignants afin d'aider les élèves à transcrire les mots. En effet, les élèves apprennent très tôt à segmenter la chaine sonore, la plupart d'entre eux étant en mesure de scander les syllabes d'un mot prononcé par un adulte en fin de moyenne section. Les pratiques pédagogiques sont alors centrées sur l'oral. L'écrit interfère peu dans la mesure où les compétences visées en fin d'école maternelle se limitent à l'écriture cursive du prénom sans modèle et à l'écriture autonome d'un seul mot "en utilisant des lettres ou groupes de lettres empruntés aux mots connus » (Ministère de l'Éducation nationale, 2020). Au cours préparatoire, les enseignants hésitent parfois entre la segmentation syllabique de l'oral utilisée à l'école maternelle et un autre type de segmentation dit « écrite ». La première semble pourtant la plus appropriée dans la mesure où elle permet de s'interroger sur le 
rôle du « e » en finale du mot « cabane » par exemple, celui-ci se prononçant /kabã/ en son absence. Elle évite également la transformation de la valeur du «e " diacritique, l'insistance sur la dernière syllabe du mot «cabane " conduisant à ce qu'elle soit prononcée /nə/.

En conclusion, la pratique de la dictée n'induit pas nécessairement une meilleure maitrise de l'orthographe, notamment lorsque les unités linguistiques proposées sont des syllabes. En effet, les élèves initialement faibles développent d'abord des compétences en décodage. En revanche, les durées d'enseignement de la morphologie jouent un rôle essentiel dans l'acquisition de l'orthographe. Les enseignants pourraient y être davantage sensibilisés pendant leur formation initiale et continue, la conscience morphologique étant un bon prédicteur des apprentissages ultérieurs en lecture et en écriture. Enfin, une réflexion pourrait être conduite sur le statut accordé à l'erreur, le développement de la vigilance orthographique ou encore les régularités graphotactiques, c'est-à-dire les associations de graphèmes les plus fréquents qu'il faudrait privilégier lors de l'écriture de mots inconnus. Les régularités orthographiques faisant le plus souvent l'objet d'un apprentissage implicite gagneraient donc à être explicitement enseignées.

\section{BIBLIOGRAPHIE}

Alves Martins, M., Albuquerque, A., Salvador, L. \& Silva, C. (2013). The impact of invented spelling on early spelling and reading. Journal of Writing Research, 5 (2), p. 215-237.

Alves Martins, M., Salvador, L., Albuquerque, A. \& Silva, C. (2014). Invented spelling activities in small groups and early spelling and reading. Educational Psychology, 36, p. 738-752. En ligne : http://dx.doi.org/10.1080/01443410.2014.950947

Bégin, C., Saint-Laurent, L. \& Giasson, J. (2005). La contribution des écritures provisoires dans la réussite en orthographe : étude longitudinale. Revue Canadienne de Linguistique Appliquée / CJAL. 8.2 , p. $147-166$.

Bégin, C., Saint-Laurent, L. \& Giasson, J. (2010). Le traitement morphologique dans l'écriture des mots chez les élèves de $6^{\mathrm{e}}$ année du primaire. L'année psychologique, 110, p. 275-297.

Bressoux, P. (2010). Méthodes en sciences humaines. Modélisation statistique appliquée aux sciences sociales. Louvain-la-Neuve : De Boeck.

Brissaud, C. \& Cogis, D. (2011). Comment enseigner l'orthographe aujourd'hui ? Hatier.

Brissaud, C., Pasa, L., Ragano, S. \& Totereau, C. (2016). Effets des pratiques d'enseignement de l'écriture en cours préparatoire. Revue française de pédagogie, 3 (3), p. 85-100. En ligne : https:// doi.org/10.4000/rfp.5079

Castles, A., Rastle, K. \& Nation, K. (2018). Ending the reading wars: Reading acquisition from novice to expert. Psychological Science in the Public Interest, 19, p. 5-51. En ligne :

https://doi.org/10.1177/1529100618772271 
Catach, N. (2012). L'orthographe française: L'orthographe en leçons : un traité théorique et pratique. Paris : A. Colin.

Chiss, J.-L. \& David, J. (2011). « Orthographe et production de texte ». Le français aujourd'hui, 5, p. 201-214. En ligne : http://dx.doi.org/10.3917/lfa.hs01.0201

Cogis, D., Fisher, C. \& Nadeau, M. (2015). « Quand la dictée devient un dispositif d'apprentissage ». Glottopol, 26, p. 69-91. http://glottopol.univ-rouen.fr/telecharger/numero_26/ gpl26_04cogis_fisher_nadeau.pdf

Cogis, D. (2005). Pour enseigner et apprendre l'orthographe. Nouveaux enjeux - Pratiques nouvelles. Paris : Delagrave.

Ducard, D., Honvault, R. \& Jaffré, J.-P. (1995). L'orthographe en trois dimensions. Paris : Nathan.

Fayol, M. \& Jaffré, J.-P. (2008). Orthographier. Paris : Presses universitaires de France.

Fayol, M. \& Jaffré, J.-P. (2014). « Apprendre et utiliser l'orthographe lexicale ». in : Fayol M. (dir.), L'orthographe. Paris : Presses universitaires de France, p. 55-89.

Fischer, C. \& Nadeau, M. (2014). « Usage du métalangage et des manipulations syntaxiques au cours de dictées innovantes dans des classes du primaire », Repères, 49, p. 169-191.

Goigoux, R. (dir.) (2016). Étude de l'influence des pratiques d'enseignement de la lecture et de l'écriture sur la qualité des premiers apprentissages (Rapport de recherche). En ligne : http://ife.ens-lyon.fr/ ife/recherche/lireecrire/rapport/rapport

Jaffré, J.-P. (2005). L'orthographe du français, une exception ? Le français aujourd'hui, 148 (1), p. 23-31. En ligne : « https://doi.org/10.3917/lfa.148.0023

Jaffré, J. (2006). « Pourquoi distinguer les homophones ? » Langue française, 151 (3), p. 25-40. En ligne : https://doi.org/10.3917/lf.151.0025

Ministère de l'Éducation nationale (2017). « La dictée au CP ». Ressources Éduscol. En ligne : https://cache.media.eduscol.education.fr/file/Reussite/41/5/

RA16_C2_FRA_DicteeCP_843415.pdf

Ministère de l'Éducation nationale (2019). « Pour enseigner la lecture et l'écriture au CP ». Ressources Éduscol. En ligne : « https://cache.media.eduscol.education.fr/file/Actualites/23/2/ Lecture_ecriture_versionWEB_939232.pdf.

Ministère de l'Éducation nationale (2020). Programme du cycle 2 en vigueur à la rentrée 2020 ». Bulletin officiel de l'éducation nationale $n^{\circ} 31$ du 30 juillet 2020. En ligne : https:// cache.media.eduscol.education.fr/file/A-Scolarite_obligatoire/24/5/ Programme2020_cycle_2_comparatif_1313245.pdf.

Morin, M.-F. \& Montésinos-Gelet, I. (2007). « Effet d'un programme d'orthographes approchées sur les performances ultérieures en lecture et en écriture d'élèves à risque ». Revue des sciences de l'éducation, 33 (3), p. 663-683. En ligne : http://dx.doi.org/10.7202/018963ar.

Pacton, S. (2008). «L'apprentissage de l'orthographe du français ». in : Desrochers A., Martineau F. \& Morin Y.-C. (dirs). Normes et pratiques orthographiques. Ottawa : David, p. 331-354. Rey-Debove, J. (1984). « Le domaine de la morphologie lexicale ». Cahiers de lexicologie, 45, p. 3-19. Riou, J. (2017). « Les effets du temps passé à encoder sur les performances des élèves ». Repères, 55, p. 159-182. En ligne : http://dx.doi.org/10.4000/reperes.1163.

Sautot, J.-P. (2015). « La dictée, un exercice ? » La lettre de l'AIRDF, Association internationale de recherche en didactique $\mathrm{du}$ français, p. 25-33. 
Sénéchal, M., Ouellette, G., Pagan S. \& Lever, R. (2012). « The role of invented spelling on learning to read in low-phoneme-awareness kindergartners: A randomized-control-trial study ». Reading and Writing, 25 (4), p. 917-934. En ligne : 10.1007/s11145-011-9310-2.

Séguin, H. (1974). « Morphologie grammaticale du français écrit ». in : Histoire et structure de l'orthographe française : actes du colloque international Paris - Janvier 1973. Paris :

Éd. Klincksieck/Didier, p. 46-72.

Sprenger-Charolles, L. \& Colé, P. (2013). Lecture et dyslexie. Approche cognitive. Paris : Dunod.

Véronis, J. (1988). « From sound to spelling in French: Simulation on a computer ». Cahiers de Psychologie Cognitive, 8, p. 315-334.

Ziegler, J. C., Jacobs, A. M. \& Stone, G. O. (1996). « Statistical analysis of the bidirectional inconsistency of spelling and sound in French ». Behavior Research Methods, Instruments \& Computers, 28, p. 504-515. En ligne : https://doi.org/10.3758/BF03200539.

\section{ANNEXES}

\section{Annexe 1. Classes ordonnées selon leur efficacité en orthographe}

\begin{tabular}{|l|l|l|l|l|l|l|l|}
\hline $\begin{array}{l}\text { Identifiant } \\
\text { Classe }\end{array}$ & Rang & $\begin{array}{l}\text { Coefficient } \\
\text { de } \\
\text { régression }\end{array}$ & $\begin{array}{l}\text { Significativité } \\
\text { de la variable }\end{array}$ & $\begin{array}{l}\text { Identifiant } \\
\text { Classe }\end{array}$ & Rang & $\begin{array}{l}\text { Coefficient } \\
\text { de } \\
\text { régression }\end{array}$ & $\begin{array}{l}\text { Significativité } \\
\text { de la variable }\end{array}$ \\
\hline 8 & 1 & 1,478076 & 0 & 67 & 66 & 0,3729271 & 0,139 \\
\hline $\mathbf{6 1}$ & 2 & 1,350532 & 0 & 118 & 67 & 0,3706058 & 0,172 \\
\hline 57 & 3 & 1,185153 & 0 & 107 & 68 & 0,3595371 & 0,158 \\
\hline $\mathbf{8 8}$ & 4 & 1,11861 & 0 & 110 & 69 & 0,3593638 & 0,184 \\
\hline $\mathbf{7 8}$ & 5 & 1,117764 & 0 & 125 & 70 & 0,3581756 & 0,159 \\
\hline 23 & 6 & 1,045227 & 0 & 131 & 71 & 0,3453846 & 0,189 \\
\hline $\mathbf{2 6}$ & 7 & 1,038941 & 0 & 117 & 72 & 0,2982657 & 0,249 \\
\hline $\mathbf{6 3}$ & 8 & 0,9913269 & 0 & 91 & 73 & 0,2956005 & 0,254 \\
\hline 5 & 9 & 0,9625259 & 0 & 74 & 74 & 0,2834677 & 0,298 \\
\hline 95 & 10 & 0,9447074 & 0 & 98 & 75 & 0,2815061 & 0,269 \\
\hline 85 & 11 & 0,931137 & 0 & 83 & 76 & 0,2744266 & 0,345 \\
\hline 27 & 12 & 0,9220739 & 0,001 & 3 & 77 & 0,2743406 & 0,267 \\
\hline 99 & 13 & 0,8821495 & 0,001 & 2 & 78 & 0,2709616 & 0,348 \\
\hline 53 & 14 & 0,8759062 & 0 & 10 & 79 & 0,2458206 & 0,384 \\
\hline 49 & 15 & 0,8534808 & 0,001 & 80 & 0,2445553 & 0,344 \\
\hline 25 & 16 & 0,8154549 & 0,001 & 81 & 0,2415936 & 0,339 \\
\hline 44 & 17 & 0,8070028 & 0,003 & 82 & 0,2126436 & 0,392 \\
\hline
\end{tabular}




\begin{tabular}{|c|c|c|c|c|c|c|c|}
\hline 112 & 18 & 0,8013353 & 0,002 & 97 & 83 & 0,1959343 & 0,455 \\
\hline 69 & 19 & 0,7950179 & 0,002 & 35 & 84 & 0,1955432 & 0,434 \\
\hline 130 & 20 & 0,7851679 & 0,003 & 36 & 85 & 0,1827643 & 0,479 \\
\hline 46 & 21 & 0,7827067 & 0,003 & 22 & 86 & 0,1779103 & 0,477 \\
\hline 87 & 22 & 0,7799641 & 0,004 & 101 & 87 & 0,1765206 & 0,494 \\
\hline 7 & 23 & 0,7457087 & 0,003 & 9 & 88 & 0,1686404 & 0,5 \\
\hline 82 & 24 & 0,7410117 & 0,005 & 40 & 89 & 0,1676214 & 0,527 \\
\hline 55 & 25 & 0,7352208 & 0,003 & 64 & 90 & 0,1618535 & 0,553 \\
\hline 15 & 26 & 0,7149885 & 0,004 & 28 & 91 & 0,1575779 & 0,605 \\
\hline 129 & 27 & 0,7136203 & 0,041 & 77 & 92 & 0,1542174 & 0,538 \\
\hline 32 & 28 & 0,6981885 & 0,018 & 100 & 93 & 0,1444121 & 0,593 \\
\hline 17 & 29 & 0,6979839 & 0,012 & 34 & 94 & 0,1352022 & 0,61 \\
\hline 79 & 30 & 0,6947145 & 0,009 & 86 & 95 & 0,1238905 & 0,749 \\
\hline 29 & 31 & 0,6940232 & 0,007 & 123 & 96 & 0,0954571 & 0,719 \\
\hline 47 & 32 & 0,6809076 & 0,015 & 102 & 97 & 0,0944988 & 0,714 \\
\hline 124 & 33 & 0,6631535 & 0,012 & 30 & 98 & 0,0922921 & 0,74 \\
\hline 38 & 34 & 0,6573742 & 0,013 & 106 & 99 & 0,0848073 & 0,779 \\
\hline 50 & 35 & 0,6454041 & 0,014 & 45 & 100 & 0,0841408 & 0,794 \\
\hline 43 & 36 & 0,606431 & 0,028 & 42 & 101 & 0,0801469 & 0,753 \\
\hline 65 & 37 & 0,6018478 & 0,032 & 70 & 102 & 0,0778991 & 0,78 \\
\hline 127 & 38 & 0,5916146 & 0,019 & 41 & 103 & 0,0573014 & 0,828 \\
\hline 94 & 39 & 0,5899287 & 0,027 & 128 & 104 & 0,0529413 & 0,855 \\
\hline 60 & 40 & 0,5776678 & 0,037 & 12 & 105 & 0,0511489 & 0,854 \\
\hline 80 & 41 & 0,5775768 & 0,045 & 66 & 106 & 0,0420838 & 0,873 \\
\hline 51 & 42 & 0,5745239 & 0,021 & 93 & 107 & 0,0400535 & 0,88 \\
\hline 108 & 43 & 0,5588243 & 0,037 & 37 & 108 & 0,0362182 & 0,885 \\
\hline 122 & 44 & 0,5565254 & 0,05 & 111 & 109 & 0,0322986 & 0,909 \\
\hline 114 & 45 & 0,5483779 & 0,03 & 4 & 110 & 0,0081863 & 0,979 \\
\hline 103 & 46 & 0,5413404 & 0,048 & 52 & 111 & 0,0072787 & 0,979 \\
\hline 105 & 47 & 0,5341795 & 0,097 & 96 & 112 & 0,0005332 & 0,998 \\
\hline 109 & 48 & 0,5305163 & 0,052 & 76 & 113 & $-0,0489094$ & 0,867 \\
\hline 121 & 49 & 0,5260719 & 0,037 & 68 & 114 & $-0,0541774$ & 0,844 \\
\hline 113 & 50 & 0,5220486 & 0,049 & 16 & 115 & $-0,0594638$ & 0,835 \\
\hline 84 & 51 & 0,508522 & 0,044 & 19 & 116 & $-0,0767616$ & 0,763 \\
\hline 59 & 52 & 0,5023088 & 0,058 & 90 & 117 & $-0,0932402$ & 0,729 \\
\hline
\end{tabular}




\begin{tabular}{|l|l|l|l|l|l|l|l|}
\hline 24 & 53 & 0,4999786 & 0,074 & 71 & 118 & $-0,1046201$ & 0,685 \\
\hline 89 & 54 & 0,4994148 & 0,057 & 31 & 119 & $-0,1081756$ & 0,684 \\
\hline 1 & 55 & 0,4892069 & 0,058 & 54 & 120 & $-0,1095155$ & 0,705 \\
\hline 14 & 56 & 0,486197 & 0,052 & 39 & 121 & $-0,1195576$ & 0,647 \\
\hline 33 & 57 & 0,4653709 & 0,079 & 58 & 122 & $-0,1382812$ & 0,648 \\
\hline 126 & 58 & 0,463468 & 0,081 & 62 & 123 & $-0,1519944$ & 0,579 \\
\hline 72 & 59 & 0,4387754 & 0,148 & 56 & 124 & $-0,1560777$ & 0,542 \\
\hline 11 & 60 & 0,4260348 & 0,125 & 81 & 125 & $-0,1628045$ & 0,545 \\
\hline 48 & 61 & 0,4095091 & 0,128 & $\mathbf{1 1 9}$ & 126 & $-0,1929551$ & 0,461 \\
\hline 21 & 62 & 0,3845949 & 0,134 & $\mathbf{1 0 4}$ & 127 & $-0,2128854$ & 0,415 \\
\hline 6 & 63 & 0,3834597 & 0,137 & $\mathbf{1 1 6}$ & 128 & $-0,3819999$ & 0,14 \\
\hline 75 & 64 & 0,3818573 & 0,149 & $\mathbf{9 2}$ & 129 & $-0,4242397$ & 0,109 \\
\hline 18 & 65 & 0,3773577 & 0,136 & $\mathbf{2 0}$ & 130 & $-0,4277947$ & 0,097 \\
\hline
\end{tabular}

Note de lecture : la première colonne du tableau correspond à l'identifiant des 131 classes de l'étude Lire et Écrire. La seconde indique le rang de chacune de ces classes selon son efficacité en orthographe. La troisième précise les valeurs des coefficients du modèle de régression et la quatrième, la significativité de l'effet d'appartenance à une classe. Les identifiants qui apparaissent en gras dans la première colonne sont ceux des classes dont nous avons exploité les enregistrements vidéo.

L'efficacité des classes a été déterminée à l'aide d'une analyse de régression dont le but est d'expliquer la variété des performances finales des élèves en orthographe. Afin d'isoler l'effet spécifique des classes, cinq facteurs individuels affectant la progression des élèves ont été contrôlés : le niveau initial des élèves, leur sexe, leur âge, la (ou les) langue(s) parlée(s) à la maison et la catégorie socio-professionnelle des parents. La classe 120 a servi de référence, elle n'apparait donc pas dans le tableau.

\section{Annexe 2. Typologie des tâches}

\section{PG : Phono - graphie}

PG1. Étudier les phonèmes (sans écrit)

PG2. Étudier les syllabes orales ou d'autres unités de taille supérieure au phonème (rime) (sans écrit)

PG3. Étudier les lettres (noms ; différentes écritures ; sans valeur sonore)

PG4. Étudier les correspondances entre phonèmes et graphèmes (CGP)

PG5. Étudier la combinatoire ou travailler sur les syllabes

\section{L : Lecture}

L1. Lire silencieusement [M P T]

L2. Reconnaitre un mot entier 
L3. Déchiffrer un mot

L4. Lire à haute voix $[\mathrm{P} \mathrm{T}]$

L5. Écouter la maitresse / le maitre lire à haute voix [P T]

EL : Étude de la langue

EL1. Lexique (sens et construction des mots)

EL2. Syntaxe (ordre des mots et valeur des mots outils)

EL3. Morphologie (orthographe des mots, chaine des accords et désinences verbales)

É : Écriture

E1. Calligraphier [L S M]

E2. Copier (avec modèle) [L S M P T]

E3. Copier après disparition du modèle [L S M P T] (copie différée)

E4. Écrire sous la dictée [L S M P T] (Le maître décide des unités à écrire)

E5. Produire en combinant des unités linguistiques déjà imprimées [S M P T] (coder l'unité à produire)

E6. Produire en dictant à autrui [L S M P T]

E7. Produire en encodant soi-même [S M P T] (Les élèves choisissent)

E8. Définir, planifier ou organiser la tâche d'écriture (enjeu, destinataire, contenu, plan...)

E9. Revenir sur l'écrit produit : le commenter, le corriger, le réviser, l'améliorer.

[L S M P T] Préciser sur quel type d'unité linguistique porte la tâche :

$\mathrm{L}=$ Lettre $; \mathrm{S}=$ Syllabe $; \mathrm{M}=\operatorname{Mot} ; \mathrm{P}=$ Phrase $; \mathrm{T}=$ Texte

\section{Annexe 3. Modalités d'organisation pédagogique et spécificités didactiques des classes les plus efficaces}

\begin{tabular}{|l|l|l|l|l|l|l|l|}
\hline $\begin{array}{l}\text { Eff. } \\
\text { (Rang) }\end{array}$ & $\begin{array}{l}\text { Id. } \\
\text { classe }\end{array}$ & $\begin{array}{l}\text { Nature de la } \\
\text { séance et } \\
\text { durée }\end{array}$ & $\begin{array}{l}\text { Tâches } \\
\text { prescrite }\end{array}$ & $\begin{array}{l}\text { Choix des } \\
\text { unités } \\
\text { linguistiques } \\
\text { dictées }\end{array}$ & Supports & $\begin{array}{l}\text { Regroupements } \\
\text { d'élèves }\end{array}$ & $\begin{array}{l}\text { Spécificités } \\
\text { didactiques }\end{array}$ \\
\hline
\end{tabular}




\begin{tabular}{|c|c|c|c|c|c|c|c|}
\hline 2 & 61 & $\begin{array}{l}\text { Dictée de } \\
\text { syllabes ( } 9 \text { min } \\
42 \text { ) }\end{array}$ & $\begin{array}{l}\text { E4-S, E6- } \\
\text { L, PG4, } \\
\text { EL3 }\end{array}$ & $\begin{array}{l}\text { « poi », } \\
\text { « toi », } \\
\text { « moi », } \\
\text { « roi » }\end{array}$ & $\begin{array}{l}\text { Ardoise, } \\
\text { tableau } \\
\text { pour la } \\
\text { correction } \\
\text { des } \\
\text { syllabes }\end{array}$ & $\begin{array}{l}\text { Collectif, } \\
\text { recherches } \\
\text { individuelles }\end{array}$ & $\begin{array}{l}\text { La correction se } \\
\text { déroule } \\
\text { immédiatement } \\
\text { après la dictée } \\
\text { de chacune des } \\
\text { syllabes. Les } \\
\text { propositions } \\
\text { des élèves sont } \\
\text { discutées avant } \\
\text { que la forme } \\
\text { correcte soit } \\
\text { validée par } \\
\text { l'enseignant. }\end{array}$ \\
\hline 3 & 57 & $\begin{array}{l}\text { Dictée de mots } \\
(19 \min 42)\end{array}$ & $\begin{array}{l}\text { E4-M, } \\
\text { E9, E6-L }\end{array}$ & $\begin{array}{l}\text { " chapiteau ", } \\
\text { « voile ", } \\
\text { « biberon », } \\
\text { " baleine », } \\
\text { " fourmi ", } \\
\text { « bouton" }\end{array}$ & $\begin{array}{l}\text { Ardoise, } \\
\text { tableau } \\
\text { pour la } \\
\text { correction } \\
\text { des mots }\end{array}$ & $\begin{array}{l}\text { Collectif, } \\
\text { recherches } \\
\text { individuelles }\end{array}$ & $\begin{array}{l}\text { En cours } \\
\text { d'écriture, } \\
\text { l'enseignante } \\
\text { propose des } \\
\text { mots de } \\
\text { référence afin } \\
\text { que les élèves } \\
\text { opèrent les } \\
\text { bons choix } \\
\text { parmi plusieurs } \\
\text { phonogrammes. }\end{array}$ \\
\hline 4 & 88 & $\begin{array}{l}\text { Dictée de } \\
\text { syllabes et } \\
\text { d'une phrase } \\
(17 \text { min } 29)\end{array}$ & $\begin{array}{l}\text { E4-S, E4- } \\
\text { P, E6-L, } \\
\text { E9 }\end{array}$ & $\begin{array}{l}\text { «bou », } \\
\text { "pa », } \\
\text { «bul », } \\
\text { «bé », } \\
\text { «bro », } \\
\text { «blu » « Le } \\
\text { bébé est dans } \\
\text { les bras de } \\
\text { maman » }\end{array}$ & \begin{tabular}{|l|} 
Cahier, \\
tableau \\
pour la \\
correction \\
des \\
syllabes et \\
de la \\
phrase
\end{tabular} & $\begin{array}{l}\text { Collectif, } \\
\text { recherches } \\
\text { individuelles }\end{array}$ & $\begin{array}{l}\text { L'enseignante } \\
\text { propose aux } \\
\text { élèves des mots } \\
\text { référents qui } \\
\text { comportent les } \\
\text { syllabes dictées, } \\
\text { par exemple } \\
\text { «c'est le bou de } \\
\text { bouchon » ou } \\
\text { "c'est le bé de } \\
\text { bébé ». }\end{array}$ \\
\hline
\end{tabular}




\begin{tabular}{|c|c|c|c|c|c|c|c|}
\hline 5 & 78 & $\begin{array}{l}\text { Dictée de } \\
\text { groupes } \\
\text { nominaux (11 } \\
\text { min14) }\end{array}$ & E4-M & $\begin{array}{l}\text { « deux } \\
\text { filles ", } \\
\text { " quatre } \\
\text { boutons ", } \\
\text { " sept } \\
\text { bananes ", } \\
\text { " cinq } \\
\text { garçons », } \\
\text { "six } \\
\text { camions », } \\
\text { "trois vélos » }\end{array}$ & Cahier & $\begin{array}{l}\text { Travail } \\
\text { individuel }\end{array}$ & $\begin{array}{l}\text { L'enseignante a } \\
\text { intitulé sa } \\
\text { séance « dictée } \\
\text { muette ». En } \\
\text { tout début de } \\
\text { séance, elle } \\
\text { donne aux } \\
\text { élèves des } \\
\text { indications sur } \\
\text { les marques du } \\
\text { pluriel. Puis les } \\
\text { élèves } \\
\text { travaillent } \\
\text { seuls. Il n'y a } \\
\text { pas de } \\
\text { correction } \\
\text { immédiate de la } \\
\text { dictée. }\end{array}$ \\
\hline 7 & 26 & $\begin{array}{l}\text { Dictée de } \\
\text { syllabes, de } \\
\text { mots et d'une } \\
\text { phrase autour } \\
\text { du phonème / } \\
\text { g/ (48 min) }\end{array}$ & $\begin{array}{l}\text { E4-S, E4- } \\
\text { M, E6-L }\end{array}$ & 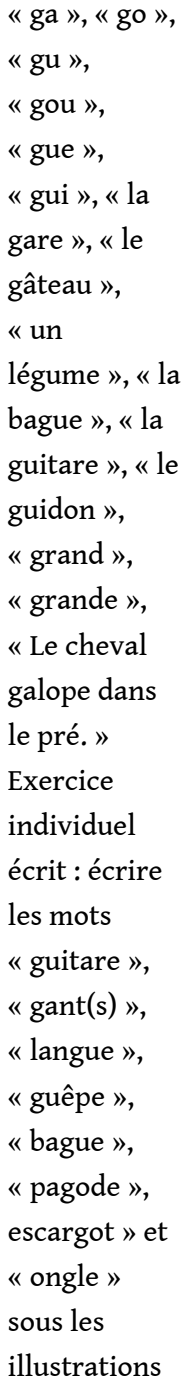 & $\begin{array}{l}\text { Tableau, } \\
\text { feuille } \\
\text { volante }\end{array}$ & & $\begin{array}{l}\text { De nombreux } \\
\text { échanges } \\
\text { portent soit sur } \\
\text { l'écriture des } \\
\text { lettres finales, } \\
\text { soit sur } \\
\text { l'écriture de } \\
\text { graphèmes } \\
\text { lorsque } \\
\text { plusieurs } \\
\text { transcriptions } \\
\text { sont possibles. }\end{array}$ \\
\hline
\end{tabular}




\begin{tabular}{|c|c|c|c|c|c|c|c|}
\hline 8 & 63 & $\begin{array}{l}\text { Dictée de } \\
\text { groupes } \\
\text { nominaux, } \\
\text { d'un groupe } \\
\text { prépositionnel, } \\
\text { d'un verbe et } \\
\text { d'une phrase } \\
\text { (32 min 19) }\end{array}$ & $\begin{array}{l}\text { E4-M, } \\
\text { E4-P }\end{array}$ & $\begin{array}{l}\text { " la tortue ", } \\
\text { " une tache ", } \\
\text { "sur la } \\
\text { route », « une } \\
\text { tomate », } \\
\text { " une } \\
\text { bouche », } \\
\text { " rire », « il a } \\
\text { lu le menu» }\end{array}$ & Cahier & & $\begin{array}{l}\text { L'enseignante } \\
\text { donne des } \\
\text { précisions sur } \\
\text { l'écriture des } \\
\text { mots au fur et à } \\
\text { mesure qu'elle } \\
\text { les dicte, } \\
\text { notamment sur } \\
\text { la présence de } \\
\text { lettres muettes. } \\
\text { Elle considère } \\
\text { qu'il faut écrire } \\
\text { ce qu'on } \\
\text { entend. }\end{array}$ \\
\hline 15 & 49 & $\begin{array}{l}\text { Dictée de } \\
\text { groupes } \\
\text { nominaux (21 } \\
\text { min) }\end{array}$ & $\begin{array}{l}\text { E4-S, } \\
\text { E4-M }\end{array}$ & $\begin{array}{l}\text { /kAR/, /ku/, } \\
\text { /k9/ /kap/, / } \\
\mathrm{kol} /, / \\
\mathrm{kyb}(\mathrm{e}) /, / \\
\mathrm{kHi} /, / \mathrm{kAl} /, / \\
\mathrm{ko} /, / \mathrm{ku} /, \\
\text { « une } \\
\text { gomme », } \\
\text { « de la } \\
\text { colle », } \\
\text { «l'école », } \\
\text { « un carré », } \\
\text { « une } \\
\text { cabane », } \\
\text { « un cube », } \\
\text { « une robe », } \\
\text { « une } \\
\text { corde », « un } \\
\text { carton », « un } \\
\text { coude » }\end{array}$ & Cahier & $\begin{array}{l}\text { Travail } \\
\text { individuel }\end{array}$ & $\begin{array}{l} \\
\text { L'enseignante } \\
\text { donne des } \\
\text { indications sur } \\
\text { les lettres } \\
\text { muettes en } \\
\text { finale, elle } \\
\text { prononce les } \\
\text { syllabes écrites } \\
\text { afin d'aider les } \\
\text { élèves. Il n'y a } \\
\text { pas de } \\
\text { correction } \\
\text { immédiate de la } \\
\text { dictée. }\end{array}$ \\
\hline
\end{tabular}

\section{Annexe 4. Modalités d'organisation pédagogique et spécificités didactiques des classes les moins efficaces}

\begin{tabular}{|l|l|l|l|l|l|l|l|}
\hline $\begin{array}{l}\text { Eff. } \\
\text { (Rang) }\end{array}$ & $\begin{array}{l}\text { Id. } \\
\text { classe }\end{array}$ & $\begin{array}{l}\text { Nature de la } \\
\text { séance et } \\
\text { durée }\end{array}$ & $\begin{array}{l}\text { Tâches } \\
\text { prescrite }\end{array}$ & $\begin{array}{l}\text { Choix des unités } \\
\text { linguistiques } \\
\text { dictées }\end{array}$ & Supports & $\begin{array}{l}\text { Regroupements } \\
\text { d'élèves }\end{array}$ & $\begin{array}{l}\text { Spécificités } \\
\text { didactiques }\end{array}$ \\
\hline
\end{tabular}




\begin{tabular}{|c|c|c|c|c|c|c|c|}
\hline 122 & 58 & $\begin{array}{l}\text { Écriture de } \\
\text { syllabes et } \\
\text { reconstitution } \\
\text { de mots } \\
\text { (syllabes } \\
\text { fournies) (26 } \\
\text { min) }\end{array}$ & $\begin{array}{l}\text { E4-S, E4- } \\
\text { M }\end{array}$ & 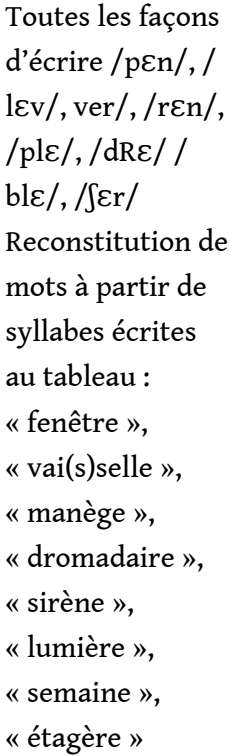 & $\begin{array}{l}\text { Ardoise et } \\
\text { tableau }\end{array}$ & $\begin{array}{l}\text { Travail } \\
\text { individuel }\end{array}$ & $\begin{array}{l}\text { L'enseignante } \\
\text { écrit au } \\
\text { tableau les } \\
\text { graphies } \\
\text { proposées la } \\
\text { plupart du } \\
\text { temps sans les } \\
\text { épeler ou sans } \\
\text { les faire } \\
\text { épeler. } \\
\text { L'exercice } \\
\text { d'écriture de } \\
\text { mots a peu de } \\
\text { lien avec les } \\
\text { syllabes } \\
\text { écrites } \\
\text { précédemment }\end{array}$ \\
\hline
\end{tabular}




\begin{tabular}{|c|c|c|c|c|c|c|}
\hline 126 & 119 & $\begin{array}{l}\text { Dictée d'une } \\
\text { phrase (10 } \\
\text { min) }\end{array}$ & E4-P & 要 & $\begin{array}{l}\text { Cahier + } \\
\text { tableau }\end{array}$ & $\begin{array}{l}\text { Le maitre } \\
\text { prévoit un } \\
\text { certain nb } \\
\text { d'aides } \\
\text { (matérialisation } \\
\text { lettres et } \\
\text { mots, précise } \\
\text { que les élèves } \\
\text { ont les aides à } \\
\text { leur } \\
\text { disposition } \\
\text { dans la } \\
\text { classe). Il a } \\
\text { tendance à } \\
\text { faire les } \\
\text { questions et } \\
\text { les réponses, } \\
\text { s'appuie peu } \\
\text { sur ce qu'ont } \\
\text { écrit les } \\
\text { élèves (« vous } \\
\text { allez récrire } \\
\text { au-dessous la } \\
\text { phrase du } \\
\text { tableau non } \\
\text { pas en } \\
\text { regardant ce } \\
\text { que vous avez } \\
\text { écrit mais en } \\
\text { regardant ce } \\
\text { qu'il y a au } \\
\text { tableau ») et } \\
\text { les élèves } \\
\text { recopient la } \\
\text { dictée écrite } \\
\text { au tableau par } \\
\text { le maitre } \\
\text { " pour les } \\
\text { parents »! } \\
\text { (« Je vais vous } \\
\text { dicter la } \\
\text { phrase et } \\
\text { ensuite on va } \\
\text { la copier aux } \\
\text { parents »). }\end{array}$ \\
\hline
\end{tabular}




\begin{tabular}{|c|c|c|c|c|c|c|c|}
\hline 127 & 104 & $\begin{array}{l}\text { Dictée de } \\
\text { syllabes et } \\
\text { d'une phrase } \\
\text { (13 min) }\end{array}$ & $\begin{array}{l}\text { E4-S, E4- } \\
\text { M, E4-P }\end{array}$ & $\begin{array}{l}\text { « tes », « chez », } \\
\text { « les », « ré », } \\
\text { « vez » « l'école, } \\
\text { « un sorcier », } \\
\text { « le nez », } \\
\text { « mes » « Le } \\
\text { sorcier va tuer } \\
\text { assez } \\
\text { d'araignées.» }\end{array}$ & $\begin{array}{l}\text { Tableau + } \\
\text { cahier }\end{array}$ & $\begin{array}{l}\text { Correction } \\
\text { collective. Un } \\
\text { élève est } \\
\text { envoyé au } \\
\text { tableau pour } \\
\text { écrire un seul } \\
\text { mot à la fois (y } \\
\left.\text { compris } d^{\prime}\right) \text {. }\end{array}$ & $\begin{array}{l}\text { Correction de } \\
\text { la dictée : } \\
\text { l'enseignant } \\
\text { dicte mot par } \\
\text { mot, y } \\
\text { compris les } \\
\text { mots de la } \\
\text { phrase (non } \\
\text { relue en } \\
\text { entier). Une } \\
\text { seule graphie } \\
\text { attendue pour } \\
\text { les syllabes. }\end{array}$ \\
\hline 128 & 116 & $\begin{array}{l}\text { Dictée de } \\
\text { mots épelés et } \\
\text { tracés dans } \\
\text { l'espace ( } 9 \\
\text { min) }\end{array}$ & E4-M & $\begin{array}{l}\text { «Dans », « les », } \\
\text { « pas », « je », } \\
\text { « il », « elle» }\end{array}$ & Ardoise & & $\begin{array}{l}\text { Apparemment } \\
\text { la première } \\
\text { dictée (sur } \\
\text { ardoise), non } \\
\text { présentée } \\
\text { comme telle } \\
\text { ( u une } \\
\text { nouvelle } \\
\text { petite activité } \\
\text { qu'on va avoir } \\
\text { ensemble et } \\
\text { qu'on va } \\
\text { continuer } \\
\text { jusqu'à la fin } \\
\text { de l'année »). } \\
\text { Les enfants } \\
\text { vont écrire } 5 \\
\text { mots. On va } \\
\text { faire plusieurs } \\
\text { petits jeux ». } \\
\text { De fait tous } \\
\text { les élèves } \\
\text { écrivent les } \\
\text { mots choisis. } \\
\text { Il va falloir } \\
\text { que vous } \\
\text { sachiez les } \\
\text { écrire sans les } \\
\text { regarder... } \\
\text { mais avant on } \\
\text { va } \\
\text { s'entrainer.» }\end{array}$ \\
\hline
\end{tabular}




\begin{tabular}{|c|c|c|c|c|c|c|}
\hline 129 & 92 & $\begin{array}{l}\text { Dictée de } \\
\text { syllabes et } \\
\text { d'une phrase } \\
(24 \mathrm{~min})\end{array}$ & $\begin{array}{l}\text { E4-S, E4- } \\
\text { P, L4-P }\end{array}$ & \begin{tabular}{|l} 
\\
\\
\\
«pa », «ru $/$ \\
«lon », «prou» \\
$/$ « ni », «plen » \\
$/$ «tou », «tru » \\
« Mon papa \\
mange une \\
pomme. »
\end{tabular} & $\begin{array}{l} \\
\\
\text { Cahier, } \\
\text { une } \\
\text { tablette } \\
\text { (sur } \\
\text { laquelle } \\
\text { les élèves } \\
\text { écrivent } \\
\text { tour à } \\
\text { tour), } \\
\text { tableau } \\
\text { numérique } \\
\text { pour la } \\
\text { correction }\end{array}$ & $\begin{array}{l}\text { L'enseignant } \\
\text { insiste sur la } \\
\text { façon de s'y } \\
\text { prendre pour } \\
\text { écrire une } \\
\text { syllabe, pour } \\
\text { recopier un } \\
\text { mot (« un mot } \\
\text { long on le } \\
\text { découpe en } \\
\text { syllabes »). } \\
\text { Rythme très } \\
\text { lent (12 min } \\
\text { sur les } \\
\text { syllabes). La } \\
\text { phrase de la } \\
\text { dictée peut } \\
\text { être projetée } \\
\text { au préalable } \\
\text { au tableau } \\
\text { blanc et } \\
\text { l'enseignant a } \\
\text { le souci d'en } \\
\text { faciliter la } \\
\text { mémorisation. }\end{array}$ \\
\hline 130 & 20 & $\begin{array}{l}\text { Dictée de } \\
\text { mots incluant } \\
\text { le son } / \mathrm{b} / 20 \\
\text { min }+ \\
\text { correction (9 } \\
\text { min } 30 \text { pour } \\
\text { dicter les } 2 \\
\text { premiers } \\
\text { mots) }\end{array}$ & & $\begin{array}{l}\text { « robe», } \\
\text { «cabane ", } \\
\text { «baraque » }\end{array}$ & $\begin{array}{l}\text { Cahier } \\
\text { (tableau) }\end{array}$ & $\begin{array}{l}\text { L'aide } \\
\text { apportée : le } \\
\text { nombre de } \\
\text { syllabes } \\
\text { écrites est } \\
\text { matérialisé au } \\
\text { tableau. } \\
\text { /Ro/ et / bə/ }\end{array}$ \\
\hline
\end{tabular}

\section{Annexe 5. Modélisation multiniveau}

Modèle multiniveau estimant l'effet des durées des tâches de morphologie sur les performances en orthographe des élèves de cours préparatoire $\left(\mathrm{N}=2481^{\mathrm{a}}\right)$ 
LR test vs. linear regression: $\operatorname{chibar} 2(01)=128.82$ Prob $>=$ chibar2 $=0.0000$

\begin{tabular}{|c|c|c|c|c|c|c|}
\hline Paramètres & Coefficients & $\begin{array}{l}\text { Erreur-type } \\
\text { des } \\
\text { coefficients }\end{array}$ & $z$ & $P>|z|$ & \multicolumn{2}{|c|}{$\begin{array}{l}\text { [95\% Intervalle de } \\
\text { confiance] }\end{array}$} \\
\hline \multicolumn{7}{|l|}{ Effets fixes } \\
\hline Constante & 0.0234002 & 0.1654024 & 0.14 & 0.887 & -0.3007826 & 0.347583 \\
\hline Score individuel initial & 0.3654589 & 0.0185653 & 19.69 & 0.000 & 0.3290716 & 0.4018463 \\
\hline $\begin{array}{l}\text { Fille (référence garçon) } \\
\text { Profession des parents : }\end{array}$ & 0.1404083 & 0.0339138 & 4.14 & 0.000 & 0.0739384 & 0.2068783 \\
\hline Catégorie défavorisée & -0.01826 & 0.0524724 & -0.35 & 0.728 & -0.1211039 & 0.084584 \\
\hline $\begin{array}{l}\text { Catégorie intermédiaire } \\
\text { (référence catégorie } \\
\text { fovorisée) }\end{array}$ & -0.202622 & 0.0525604 & -3.86 & 0.000 & -0.3056384 & -0.0996056 \\
\hline Âge: & -0.8160592 & 0.0894357 & -9.12 & 0.000 & -0.99135 & -0.6407684 \\
\hline Retard scolaire & -0.0516434 & 0.0430437 & -1.20 & 0.230 & -0.1360074 & 0.0327206 \\
\hline Entre mai et août & -0.0993014 & 0.0438353 & -2.27 & 0.023 & -0.185217 & -0.0133857 \\
\hline \multicolumn{7}{|l|}{$\begin{array}{l}\text { Entre sept. et déc. } \\
\text { (référence entre janv. et avril) } \\
\text { Langue(s) parlée(s) à la }\end{array}$} \\
\hline maison :Autre & -0.3078625 & 0.0742011 & -4.15 & 0.000 & -0.4532939 & -0.1624311 \\
\hline $\begin{array}{l}\text { Français + autre } \\
\text { (référence français) }\end{array}$ & -0.0034777 & 0.0481105 & -0.07 & 0.942 & -0.0977725 & 0.090817 \\
\hline $\begin{array}{l}\text { Rythme scolaire } \\
\text { (référence } 4.5 \mathrm{j} / \text { semaine) }\end{array}$ & -0.0046231 & 0.0770371 & -0.06 & 0.952 & -0.1556131 & 0.1463669 \\
\hline Éducation prioritaire & -0.0920464 & 0.0790017 & -1.17 & 0.244 & -0.246887 & 0.0627941 \\
\hline $\begin{array}{l}\text { Niveau moyen initial dans } \\
\text { l'épreuve }\end{array}$ & -0.2389112 & 0.0885469 & -2.70 & 0.007 & -0.41246 & -0.0653625 \\
\hline $\begin{array}{l}\text { Hétérogénéité initiale dans } \\
\text { l'épreuve }\end{array}$ & 0.0109391 & 0.1417241 & 0.08 & 0.938 & -0.266835 & 0.2887133 \\
\hline$\%$ PCS favorisé & 0.1909339 & 0.2031977 & 0.94 & 0.347 & -0.2073263 & 0.5891942 \\
\hline $\begin{array}{l}\text { Ancienneté au cours } \\
\text { préparatoire }\end{array}$ & -0.0034639 & 0.0060396 & -0.57 & 0.566 & -0.0153012 & 0.0083735 \\
\hline $\begin{array}{l}\text { Effet de la variable durée des } \\
\text { tâches de morphologie }\end{array}$ & 0.0044457 & 0.0010831 & 4.10 & 0.000 & 0.0023228 & 0.0065687 \\
\hline Effets aléatoires & 0.0838697 & 0.0149854 & & & 0.0590904 & 0.1190401 \\
\hline $\begin{array}{l}\text { Niveau } 2 \text { : variable interclasse } \\
\text { Niveau } 1 \text { : variable intraclasse }\end{array}$ & 0.6921025 & 0.0201847 & & & 0.6536507 & 0.7328164 \\
\hline$-2 \log L=-3141.2645$ & & & & & & \\
\hline
\end{tabular}

Note de lecture : les résultats figurant dans le tableau ci-dessus indiquent que la durée des tâches de morphologie a un effet significatif sur les performances des élèves en orthographe $(p<0.001)$. Plusieurs variables susceptibles d'expliquer les performances finales des élèves en orthographe ont été contrôlées. Les unes portent sur les caractéristiques des élèves (âge, sexe, langue(s) parlée(s) à la maison, profession des parents), les autres sur les caractéristiques de la classe (rythme scolaire, établissement appartenant ou non à l'éducation prioritaire, niveau moyen initial dans l'épreuve, hétérogénéité initiale dans l'épreuve, pourcentage de catégories socio-professionnelles favorisées, ancienneté de l'enseignant au cours préparatoire).

\section{NOTES}

1. La liste proposée par le ministère de l'Éducation nationale (2017) compte neuf dictées : dictée de syllabes, dictée de mots usuels, dictée à l'adulte, dictée à partir d'une dictée à l'adulte, dictée sans erreur (Ouzoulias, 2004), phrase dictée du jour (Brissaud \& Cogis, 2011), dictée hebdomadaire, dictée frigo, dictée négociée. https:// cache.media.eduscol.education.fr/file/Reussite/41/5/

RA16_C2_FRA_DicteeCP_843415.pdf

Notons que ces propositions mêlent des tâches de nature très différentes qui ne relèvent pas toutes de l'encodage tel que nous l'avons défini plus haut.

2. Sur les 34920 mots français répertoriés dans le dictionnaire Le Robert méthodique, 80 $\%$ sont morphologiquement complexes, c'est-à-dire constitués d'au moins deux morphèmes (Rey-Debove, 1984). 
3. Voir Annexe 2. Typologie des tâches d'écriture (extrait de la typologie des tâches de la recherche Lire et Écrire).

4. La phrase dictée par l'enseignant de la classe 104 est : «Le sorcier va tuer assez d'araignées. ». L'expression du futur proche construit avec le verbe « aller » suivi d'un infinitif, l'adverbe « assez » et le pluriel du nom " araignées » sont des difficultés qui, pour être surmontées, réclament une bonne connaissance du fonctionnement de l'orthographe grammaticale et lexicale.

5. Le terme " quadratique " renvoie à une relation non linéaire entre la variable explicative et la variable à expliquer. Celle-ci se matérialise par une courbe en $U$ ou U inversé. La courbe en U indique que l'effet de la variable explicative diminue jusqu'à un minimum puis augmente, la courbe en U inversé qu'il augmente jusqu'à un maximum puis diminue.

a. Sur les 2507 élèves ayant passé les épreuves initiales et finales, 26 ont été retirés des analyses en raison de données manquantes relatives à la profession des parents. Ces élèves appartiennent à des classes différentes et ce retrait ne modifie pas la structure de l'échantillon.

\section{RÉSUMÉS}

La traditionnelle dictée et sa fonction évaluative ont vu naitre de nouvelles versions de l'exercice visant à instaurer une vigilance orthographique et à doter les élèves de capacités à lever leurs incertitudes face à la norme. Ces approches innovantes ont parfois conduit à des évolutions dans les pratiques d'enseignement. C'est dans ce contexte que les auteurs s'attachent à caractériser les pratiques efficaces de la dictée en première primaire en France $(\mathrm{CP})$ en contrastant des classes où les élèves progressent le plus versus le moins en orthographe. Les pratiques efficaces apparaissent structurées et progressives, notamment du point de vue des unités linguistiques dictées. Elles intègrent un traitement systématique des erreurs qui prend appui sur les spécificités du système orthographique. De plus, les enseignants des classes efficaces consacrent davantage de temps que leurs pairs à l'enseignement de la morphologie, ce qui influence significativement et positivement les progrès des élèves en orthographe.

The traditional dictation and its evaluative function have seen the emergence of new versions of the exercise aimed at establishing spelling vigilance and equipping pupils with the ability to overcome their uncertainties regarding the norm. These innovative approaches have sometimes led to changes in teaching practices. It is in this context that the authors seek to characterise the effective practices of dictation in the first year of primary school in France (CP) by contrasting the classes where pupils make the most versus the least progress in spelling. The effective practices appear to be structured and progressive, particularly in terms of the linguistic units dictated. They incorporate a systematic treatment of errors based on the specific features of the spelling system. In addition, teachers in effective classes spend more time than their peers on teaching morphology, which has a significant and positive influence on pupils' progress in spelling. 
INDEX

Keywords : Spelling, dictation, efficiency, teaching, first grade

Mots-clés : Orthographe, dictée, efficacité, enseignement, cours préparatoire

\section{AUTEURS}

\section{JÉRÔME RIOU}

Université Toulouse - Jean Jaurès, EFTS, F-31000 Toulouse

\section{CATHERINE BRISSAUD}

Université Grenoble Alpes, Lidilem, F-38000 Grenoble 